PROCEEDINGS OF THE

AMERICAN MATHEMATICAL SOCIETY

Volume 132, Number 9, Pages 2713-2721

S 0002-9939(04)07402-7

Article electronically published on April 9, 2004

\title{
LOCALLY HOMOGENEOUS AFFINE CONNECTIONS ON COMPACT SURFACES
}

\author{
BARBARA OPOZDA
}

(Communicated by Jon G. Wolfson)

\begin{abstract}
Global properties of locally homogeneous and curvature homogeneous affine connections are studied. It is proved that the only locally homogeneous connections on surfaces of genus different from 1 are metric connections of constant curvature. There exist nonmetrizable nonlocally symmetric locally homogeneous affine connections on the torus of genus 1 . It is proved that there is no global affine immersion of the torus endowed with a nonflat locally homogeneous connection into $\mathbf{R}^{3}$.
\end{abstract}

The best known locally homogeneous affine connections are flat ones. J. Milnor, [3], proved that there are no flat affine connections on surfaces of genus different from 1. We prove an analogous result for nonflat locally homogeneous affine connnections. We prove, in fact, that a nonflat torsion-curvature homogeneous of order 1 connection on a compact surface of genus different from 1 must be torsionfree metric and locally symmetric; that is, it must be a Levi-Civita connection of constant curvature.

In contrast with this situation, there do exist nonlocally symmetric nonmetrizable locally homogeneous connections on the torus of genus 1 . We give a family of such connections.

Note that, unlike in the case of Riemannian structures, the class of locally homogeneous affine connections on 2-dimensional manifolds is much richer than the class of locally symmetric ones. Some results concerning local homogeneity are proved in [8, 9] and [10. A local classification of locally homogeneous connections on 2-dimensional manifolds is given in [5] and [12].

An important question in the theory of affine connections is whether a manifold equipped with a connection $\nabla$ can be realized as a hypersurface (immersed or imbedded) in a standard affine space in such a way that there exists a transversal bundle inducing $\nabla$. We study the realization problem for the torus equipped with nonflat locally homogeneous connections. Using the notion of extrinsic completeness, we prove that there is no immersion of the torus into $\mathbf{R}^{3}$ for which an induced connection is nonflat and locally homogeneous.

Received by the editors March 3, 2003 and, in revised form, June 16, 2003.

2000 Mathematics Subject Classification. Primary 53C05, 53C40.

Key words and phrases. Affine connection, local homogeneity.

(C)2004 American Mathematical Society 
1.

Throughout the paper we assume that manifolds are connected and of class $\mathcal{C}^{\infty}$. We set the following definitions.

Definition 1.1. A family $\left\{T_{\alpha}\right\}$ of tensor fields on a manifold $M$ is called pointwise homogeneous if for each two points $x, y \in M$ there exists a linear isomorphism $F: T_{x} M \rightarrow T_{y} M$ such that $F\left(\left(T_{\alpha}\right)_{x}\right)=\left(T_{\alpha}\right)_{y}$ for each $\alpha$, where $F$ also denotes the extension of $F$ to an isomorphism of the algebras of tensors.

Definition 1.2. An affine connection $\nabla$ on $M$ with curvature $R$ and torsion $T$ is called torsion-curvature homogeneous of order $r \geq 0$ if the family

$$
\left\{\nabla^{\alpha} T, \nabla^{\alpha} R\right\}_{0 \leq \alpha \leq r}
$$

is pointwise homogeneous. Analogously, $\nabla$ is curvature homogeneous of order $r \geq 0$ if the family $\left\{\nabla^{\alpha} R\right\}_{0 \leq \alpha \leq r}$ is pointwise homogeneous.

Definition 1.3. A connection $\nabla$ on $M$ is locally homogeneous if for each two points $x, y \in M$ there are a neighbourhood $U$ of $x$, a neighbourhood $V$ of $y$ and an affine transformation $f: U \rightarrow V$ such that $f(x)=y$.

A connection $\nabla$ is locally symmetric if $\nabla R=0$ and $\nabla T=0$. A locally symmetric connection is locally homogeneous. The converse is not true. Locally homogeneous connections are torsion-curvature homogeneous of any order.

For a connection $\nabla$ the image of its curvature tensor at $x \in M$ is defined as

$$
\operatorname{im} R_{x}=\operatorname{span}\left\{R(X, Y) Z: X, Y, Z \in T_{x} M\right\} .
$$

The nullity space of the curvature tensor is given by the intersection of $\operatorname{ker} R(X, Y)$ for all $X, Y \in T_{x} M$. In case of a two-dimensional manifold the nullity space at $x \in M$ is equal to ker $R(X, Y)$, where $X, Y$ is a basis of $T_{x} M$. The curvature rank of a connection $\nabla$ is equal to $\operatorname{dim} \operatorname{im} R$.

By an orbit of a 1-dimensional distribution we mean a maximal integral submanifold of the distribution.

We shall need the following theorem ([2], p. 147).

Theorem 1.4. A 1-dimensional distribution $\mathcal{D}$ on the torus $T^{2}$ either has a periodic orbit or all its orbits are everywhere dense.

We shall now provide some information concerning affine geometry of hypersurfaces. For a more detailed exposition we refer to $[6]$.

Let $f: M \rightarrow \mathbf{R}^{n+1}$ be a hypersurface, i.e., $\operatorname{dim} M=n$ and $f$ is an immersion.

From now on we assume that $\mathbf{R}^{n+1}$ is equipped with the standard affine connection, denoted by $\tilde{\nabla}$.

Let $\mathcal{N}$ be a vector bundle transversal to $f$. If $\xi$ is a nowhere vanishing section of $\mathcal{N}$ (possibly local), then $\xi$ defines the induced connection $\nabla$, the second fundamental form $h$, the shape operator $S$ and the 1-form $\tau$ according to the following formulas of Gauss and Weingarten:

$$
\begin{aligned}
\tilde{\nabla}_{X} f_{*} Y & =f_{*}\left(\nabla_{X} Y\right)+h(X, Y) \xi \\
\tilde{\nabla}_{x} \xi & =-f_{*}(S X)+\tau(X) \xi .
\end{aligned}
$$

The connection $\nabla$ depends only on the transversal bundle generated by $\xi$, not on a particular choice of a section $\xi$. 
The induced objects satisfy the fundamental equations

$$
\begin{gathered}
R(X, Y) Z=h(Y, Z) S X-h(X, Z) S Y \quad-\text { Gauss, } \\
\nabla h(X, Y, Z)+\tau(X) h(Y, Z)=\nabla h(Y, X, Z)+\tau(Y) h(X, Z) \quad \text {-Codazzi I, } \\
\nabla S(X, Y)-\tau(X) S Y=\nabla S(Y, X)-\tau(Y) S X \quad-\text { Codazzi II, } \\
h(S X, Y)-h(X, S Y)=d \tau(X, Y) \quad-\text { Ricci. }
\end{gathered}
$$

A hypersurface is called nondegenerate at $x$ if $h_{x}$ is nondegenerate as a bilinear form.

The Gauss equation implies the following equation for the Ricci tensor Ric of $\nabla$ :

$$
\operatorname{Ric}(Y, Z)=h(Y, Z) \operatorname{tr} S-h(S Y, Z) .
$$

It follows that if Ric is nondegenerate at a point $x$, then so is $h_{x}$.

The fundamental equations yield the following relations between $\nabla$ (which we regard as an intrinsic object on $M$ ) and the extrinsic objects $h, S$ and $\tau$ :

$$
\begin{aligned}
& \operatorname{im} R_{x} \subset \operatorname{im} S_{x}, \\
& \operatorname{ker} h_{x} \subset \operatorname{ker} R_{x},
\end{aligned}
$$

for any $x \in M$.

If $\operatorname{rk} h_{x}>1$, we have equality in (1.7). The condition $\operatorname{rk} h_{x}=1$ implies equality in (1.8), provided that $R_{x}$ does not vanish.

If for a manifold $M$ endowed with a torsion-free connection $\nabla$ there are an immersion $f: M \rightarrow \mathbf{R}^{n+1}$ and a transversal bundle inducing the given $\nabla$, we say that $\nabla$ can be realized on a hypersurface in $\mathbf{R}^{n+1}$. An immersion $f$ realizing $\nabla$ is called an affine immersion of $\nabla$.

If $\mathcal{D}$ is a distribution on $M$ and $X$ is a vector field, then the notation $X \in \mathcal{D}$ will mean that $X_{x} \in \mathcal{D}_{x}$ for all $x$ from the domain of $X$.

From now on we shall consider only 2-dimensional connected manifolds. In this case, $\operatorname{im} R_{x}=\operatorname{im} R_{x}(X, Y)$ and $\operatorname{ker} R_{x}=\operatorname{ker} R_{x}(X, Y)$ for any basis $X, Y$ of $T_{x} M$.

By a neighbourhood we shall always mean a connected open one.

2.

We shall need

Proposition 2.1. Any affine connection on a compact surface has a point where its Ricci tensor is symmetric.

Proof. Let $\nabla$ be a connection on a compact surface $M^{2}$. We can assume that $M^{2}$ is orientable. Let $\nabla^{o}$ be any torsion-free connection with symmetric Ricci tensor on $M^{2}$. Take the difference tensor

$$
Q_{X} Y=\nabla_{X} Y-\nabla_{X}^{o} Y
$$

Consider the 1-form

$$
\delta(X)=\operatorname{tr} Q_{X}
$$

Then

$$
2 d \delta(X, Y)=\operatorname{tr} \nabla Q(X, Y, \cdot)-\operatorname{tr} \nabla Q(Y, X, \cdot) .
$$

The curvature tensors $R$ and $R^{o}$ of $\nabla$ and $\nabla^{\circ}$ are related by

$$
R(X, Y) Z=R^{o}(X, Y) Z+\nabla Q(X, Y, Z)-\nabla Q(Y, X, Z)+Q_{X} Q_{Y} Z-Q_{Y} Q_{X} Z .
$$


Hence

$$
\operatorname{tr} R=\operatorname{tr} R^{o}+2 d \delta
$$

Since $\operatorname{tr} R^{o}=0$, we have

$$
\int \operatorname{tr} R=0
$$

It follows that $\operatorname{tr} R$ must vanish somewhere. This gives the desired condition.

We shall now prove

Theorem 2.2. Let $M^{2}$ be a compact 2-dimensional manifold of genus different from 1 , and let $\nabla$ be an affine connection on $M^{2}$. If $\nabla$ is torsion-curvature homogeneous of order 1, then it is the Levi-Civita connection of a definite metric tensor field of constant curvature. In particular, it is torsion-free and locally symmetric.

Proof. By a theorem of Hopf, $M^{2}$ does not admit any 1-dimensional distribution, and, consequently, it also does not admit any nowhere vanishing 1-form.

We shall first prove some lemmas.

Lemma 2.3. If the torsion tensor of $\nabla$ is pointwise homogeneous, then $\nabla$ is torsion-free.

Proof. Since the torsion tensor $T$ is a skew-symmetric tensor field of type $(1,2)$, its values define a 0 - or 1-dimensional vector subspace of the tangent space $T_{x} M^{2}$ for each $x \in M^{2}$. Since $T$ is pointwise homogeneous, $T$ determines a 0 - or 1-dimensional distribution on $M^{2}$.

Lemma 2.4. If $\nabla$ is curvature homogeneous of order 0 , then the Ricci tensor of $\nabla$ is a definite metric tensor field.

Proof. By Proposition 2.1 we know that the Ricci tensor is symmetric. Since $M^{2}$ does not admit any 1-dimensional distribution, the Ricci tensor cannot have rank 1 and cannot be nondegenerate indefinite. By the theorem of Milnor it also cannot vanish.

Lemma 2.5. If $C$ is a cubic form, $g$ a definite metric tensor field on $M^{2}$ and the pair $\{g, C\}$ is pointwise homogeneous, then $C$ is symmetric and $\operatorname{tr}_{g} C(X, \cdot, \cdot)=0$ for every $X$.

Proof. We first observe that

$$
\operatorname{tr}_{g} C(X, \cdot, \cdot)=0, \quad \operatorname{tr}_{g} C(\cdot, X, \cdot)=0, \quad \operatorname{tr}_{g} C(\cdot, \cdot, X)=0
$$

for any $X$. Set $\tau(X)=\operatorname{tr}_{g} C(X, \cdot, \cdot)$. The 1-form $\tau$ is pointwise homogeneous. Hence it vanishes on $M^{2}$. It follows that $\operatorname{tr}_{g} C(X, \cdot, \cdot)=0$. Similarly one gets the two other equalities. The equalities imply the symmetry of $C$. Indeed, let $X, Y$ be an orthonormal basis of $T_{x} M^{2}$ relative to $g$. We have

$$
\begin{array}{ll}
C(X, Y, Y)=-C(X, X, X), & C(Y, X, X)=-C(Y, Y, Y), \\
C(Y, X, Y)=-C(X, X, X), & C(X, Y, X)=-C(Y, Y, Y), \\
C(Y, Y, X)=-C(X, X, X), & C(X, X, Y)=-C(Y, Y, Y) .
\end{array}
$$

By comparing the terms in columns we see that $C$ is symmetric.

Recall that a Ricci-symmetric connection $\nabla$ on a 2-dimensioanl manifold is projectively flat if $\nabla$ Ric is totally symmetric. 
As an immediate consequence of Lemmas 2.32 .5 we get

Lemma 2.6. Let $M^{2}$ be a compact surface of genus different from 1, and let $\nabla$ be an affine connection on $M^{2}$. If $\nabla$ is torsion-curvature homogeneous of order 1 , then $\nabla$ is torsion-free, Ric is a definite metric tensor field on $M^{2}, \nabla$ is projectively flat and $\operatorname{tr}_{\operatorname{Ric}} \nabla \operatorname{Ric}(X, \cdot, \cdot)=0$ for every $X$.

We also have

Lemma 2.7. Let $M^{2}$ be a compact surface of genus different from $1, g$ a metric tensor field on $M^{2}$ and $C$ a symmetric cubic form satisfying $\operatorname{tr}_{g} C(X, \cdot, \cdot)=0$ for every $X$. If $C$ is pointwise homogeneous, then $C$ is constantly 0 on $M^{2}$.

Proof. Assume first that $M^{2}$ is orientable. Since $g$ must be definite, $M^{2}$ can be covered by an atlas of isothermal coordinates. Let $z=x+i y$ be an isothermal coordinate, $X=\partial_{x}$ and $Y=\partial_{y}$. Define a complex symmetric 3 -form by

$$
\Phi=(C(X, X, X)-i C(X, X, Y)) d z^{3} .
$$

This form is globally well defined on $M^{2}$. Since the Euler characteristic of $M^{2}$ is not zero, $\Phi$ must vanish somewhere. By the pointwise homogeneity it is identically zero on $M^{2}$. If $M^{2}$ is not orientable, we can lift $C$ and $g$ to the double covering space of $M^{2}$.

We can now finish the proof of Theorem 2.2

By virtue of Lemma 2.6 we can apply Lemma 2.7 to $C=\nabla$ Ric and $g=$ Ric. Hence $\nabla$ is the Levi-Civita connection for the metric tensor field Ric. The proof is completed.

An immediate consequence of Theorem 2.2 is the following.

Corollary 2.8. The only locally homogeneous connection on the sphere $S^{2}$ is the standard one.

We also have

Corollary 2.9. Let $\nabla$ be a connection on a surface $M^{2}$ of genus different from 1 , and let $f: M^{2} \rightarrow \mathbf{R}^{3}$ be an affine immersion of $\nabla$. If $\nabla$ is curvature homogeneous of order 1 , then $M^{2}$ is a topological sphere, $f$ is an imbedding and $f\left(M^{2}\right)$ is an ellipsoid.

Proof. Since $\nabla$ is automatically torsion-free and consequently torsion-curvature homogeneous of order 1, we can apply Theorem 2.2. By this theorem $\nabla$ is the Levi-Civita connection for the nondegenerate Ric. Using now the Cartan-Norden theorem (see for instance [6]), we get that $f$ is an isometric immersion relative to some scalar product $G$ (automatically definite because Ric is) on $\mathbf{R}^{3}$. Hence $f$ is an isometric immersion of a space of constant curvature into $\mathbf{R}^{3}$ equipped with $G$. It is known that $f$ is an imbedding of the sphere $S^{2}$ as a round sphere relative to $G$. Hence $f\left(M^{2}\right)$ is an ellipsoid.

We shall now give an easily constructed class of locally homogeneous connections on the torus of genus 1 .

Example 2.10. Let $X, Y$ be analytic nowhere vanishing tangent vector fields on the circle $S^{1}$. After trivially extending $X, Y$ to the vector fields, say again $X$, 
$Y$, tangent to the torus $T^{2}=S^{1} \times S^{1}$, we obtain a global analytic frame on $T^{2}$. Moreover, $[X, Y]=0$.

Let $A, B, C, D, E, F$ be real numbers. Define a torsion-free connection $\nabla$ on $T^{2}$ as follows:

$$
\nabla_{X} X=A X+B Y, \quad \nabla_{X} Y=C X+D Y, \quad \nabla_{Y} Y=E X+F Y .
$$

The connection is analytic, and covariant derivatives of its curvature tensor are given by the same formulas everywhere on $T^{2}$. By Theorem 7.4 from [4], $\nabla$ is locally homogeneous.

A straightforward computation shows that the Ricci tensor of $\nabla$ is symmetric and $\nabla$ is projectively flat.

Depending on the numbers $A, B, C, D, E, F$, the Ricci tensor of $\nabla$ can have rank 2 or 1 or 0 . By the Gauss-Bonnet theorem, among the connections with nondegenerate Ricci tensor there are no locally symmetric ones. On the torus, however, there do exist nonflat locally symmetric connections. For instance, let $C=D=0, E B \neq 0$, and $E B-A F=0$. Then the Ricci tensor of $\nabla$ has rank 1 , the connection is locally symmetric nonmetrizable, and, as we shall prove in the next section, none of the connections can be globally realized on a surface in $\mathbf{R}^{3}$. Recall that all locally symmetric connections on 2-dimensional manifolds can be locally realized on various surfaces; see [7].

3 .

We shall now study the realization problem for arbitrary locally homogeneous connections on the torus.

Recall the definition of extrinsic completeness given in [11.

Definition 3.1. Let $N$ be a manifold with a connection $\tilde{\nabla}$, and let $f: M \rightarrow N$ be an immersion. We say that $f$ is extrinsically complete if the following condition is fulfilled. Let $\gamma:(a, b) \rightarrow M$ be a curve, where $b \in \mathbf{R}, a \in \mathbf{R}$ or $a=-\infty$. If $f \circ \gamma$ is a geodesic in $N$ relative to $\tilde{\nabla}$, then $\lim _{t \rightarrow b} \gamma(t)$ exists in $M$.

We have

Proposition 3.2. Let $f: M \rightarrow N$ be an immersion and $\tilde{\nabla}$ a complete connection on $N$. If $M$ is compact, then $f$ is extrinsically complete.

Proof. Let $\gamma:(a, b) \rightarrow M, b \in \mathbf{R}$, be a curve such that $f \circ \gamma$ is a geodesic relative to $\tilde{\nabla}$. By the completeness of $\tilde{\nabla}$ there exists $z=\lim _{t \rightarrow b}(f \circ \gamma)(t)$ in $N$.

For any sequence $b_{n} \rightarrow b$ such that $\gamma\left(b_{n}\right)$ has a limit, say $x$ in $M$, we have $f(x)=z$. Suppose there are two sequences $b_{n} \rightarrow b, c_{n} \rightarrow b, b_{n}, c_{n} \in(a, b)$, such that $\gamma\left(b_{n}\right) \rightarrow x, \gamma\left(c_{n}\right) \rightarrow y$ and $x \neq y$. Take a neighbourhood $V$ of $x$ such that $f$ is an injection on $\bar{V}$ and almost all $\gamma\left(c_{n}\right)$ are outside $\bar{V}$. Then $\operatorname{im} \gamma \cap V$ consists of infinitely many slices of $\operatorname{im} \gamma$, and one sees that there exist points other than $x$ that are limits of $\gamma\left(d_{n}\right) \in \bar{V}$ for some $d_{n} \rightarrow b, d_{n} \in(a, b)$. This contradicts the injectivity of $f$ in $\bar{V}$. The proof is completed.

Proposition 3.3. Let $\mathcal{D}$ be a 1-dimensional distribution on the torus $T^{2}$, and let $f: T^{2} \rightarrow \mathbf{R}^{3}$ be an immersion. There is no plane field in $\mathbf{R}^{3}$ along $f$ transversal to $f_{*}(\mathcal{D})$ and constant along orbits of $\mathcal{D}$. 
Proof. Assume that $\pi$ is a plane field transversal to $f_{*}(\mathcal{D})$ and constant along the orbits of $\mathcal{D}$. If $\mathcal{D}$ has a periodic orbit, say $N$, then $N$ is a compact submanifold of $T^{2}$. Let $\langle$,$\rangle be the standard scalar product in \mathbf{R}^{3}$, and let $\mathbf{e}$ be a nonzero vector perpendicular to $\pi$ restricted to $N$. Consider the function

$$
\psi: x \rightarrow\langle f(x), \mathbf{e}\rangle \in \mathbf{R}
$$

defined along $N$. The function attains an extremum at some point, say $x \in N$. Then $\left\langle f_{*}(X), \mathbf{e}\right\rangle=0$ for every $X \in T_{x} N=\mathcal{D}_{x}$. This means that $f_{*}\left(\mathcal{D}_{x}\right) \subset \pi_{x}$, which contradicts the transversality of $\pi$ to $f_{*}(\mathcal{D})$.

If $\mathcal{D}$ has no periodic orbit, then the orbits of $\mathcal{D}$ are dense in $T^{2}$, Consequently $\pi$ is constant on $T^{2}$. Consider the function given by (3.1) defined along $T^{2}$, where $\mathbf{e}$ is a nonzero vector perpendicular to the constant plane $\pi$. At a point $x \in T^{2}$ where the function attains an extremum, we have $f_{*}\left(T_{x} T^{2}\right)=\pi_{x}$. This contradiction completes the proof.

We can now prove the main result of this section.

Theorem 3.4. Let $\nabla$ be a nonflat locally homogeneous connection on the torus $T^{2}$. There is no global affine immersion of $\nabla$ into the standard affine space $\mathbf{R}^{3}$.

Proof. Suppose $f: T^{2} \rightarrow \mathbf{R}^{3}$ is an affine immersion of $\nabla$. Note first that, by the Gauss equation and the nonflatness of $\nabla$, the second fundamental form of $f$ is nowhere zero on $T^{2}$. It follows that there is a unique transversal bundle $\mathcal{N}$ inducing the given $\nabla$. Since $T^{2}$ is orientable, there is a global nowhere vanishing section, say $\xi$, of $\mathcal{N}$. We shall use the notation introduced in Section 1.

Set $M_{1}=\{\operatorname{rk} h=1\}$ and $M_{2}=\{\operatorname{rk} h=2\}$. The curvature rank of $\nabla$ is constant on $T^{2}$. If the curvature rank is 2 , then the distribution ker $R$ is 0 -dimensional. By formula (1.8), $\operatorname{ker} h$ is also 0-dimensional. This is impossible for any immersion of the torus into $\mathbf{R}^{3}$.

From now on we assume that the curvature rank of $\nabla$ is 1 . By virtue of Theorem 3.4, we know that the Ricci tensor of $\nabla$ is symmetric. Hence $\operatorname{ker} R=\operatorname{im} R$ on $T^{2}$. Denote the 1-dimensional distribution by $\mathcal{D}$. Since $T^{2}$ is compact, the set $U$ of points where $h$ is definite is nonempty. On this set the shape operator $S$ is diagonalizable and, by (1.7) and the nondegeneracy of $h$ on $U$, rk $S=1$. Hence we have an almost product structure $(\operatorname{im} S, \operatorname{ker} S)$ on $U$. For each $x \in U$ there is a coordinate system $(u, v)$ around $x$ such that $X=\partial_{u}$ spans $\operatorname{im} S$ and $Y=\partial_{v}$ spans ker $S$. The second Codazzi equation (1.5) yields $\nabla_{Y} S X=\tau(Y) S X$. Combining this with the fact that $S X \neq 0$ and the local homogeneity of $\nabla$, we see that $\nabla$ has the property

$\left.{ }^{*}\right)$ For each $x \in T^{2}$ there is a vector field $Y$ defined around $x$ and transversal to $\mathcal{D}$ such that $\nabla_{Y} X \in \mathcal{D}$ for every $X \in \mathcal{D}$.

Assume first that the distribution $\mathcal{D}$ is not totally geodesic (automatically on the whole $T^{2}$ ). On the set int $M_{1}$ the distribution $\operatorname{ker} h$ is totally geodesic; this follows from the first Codazzi equation. On the other hand, $\operatorname{ker} h=\operatorname{ker} R$ on $M_{1}$. Therefore int $M_{1}=\emptyset$. Since $\operatorname{rk} S=1$ on $M_{2}$ and $M_{2}$ is dense in $T^{2}$, the shape operator $S$ has rank 1 everywhere on $T^{2}$. By (1.7) we have $\operatorname{im} S=\operatorname{im} R$ on $T^{2}$. The property $\left(^{*}\right)$ defines a 1-dimensional distribution, say $\tilde{\mathcal{D}}$, on $T^{2}$ determined by $Y$. Indeed, let $\tilde{Y}$ be another vector field transversal to $\mathcal{D}$ and such that $\nabla_{\tilde{Y}} X \in \mathcal{D}$ for every section $X$ of $\mathcal{D}$. Let $X$ be a nowhere vanishing section of $\mathcal{D}$ on some open 
set $V$ and $\tilde{Y}=\alpha X+\beta Y$. Then $\nabla_{\tilde{Y}} X=\alpha \nabla_{X} X+\beta \nabla_{Y} X$. Using the assumption that $\mathcal{D}$ is not totally geodesic, we obtain that $\alpha=0$.

Now define

$$
\pi=f_{*}(\mathcal{D})+\mathcal{N}
$$

where $\pi$ is a plane field transversal to $f_{*}(\tilde{\mathcal{D}})$. Observe that $\pi$ is parallel relative to $\tilde{\nabla}$ along the orbits of $\tilde{\mathcal{D}}$. This easily follows from formulas (1.1), (1.2), the equality $\operatorname{im} R=\operatorname{im} S$ and the property $(*)$. This contradicts Proposition 3.3

Assume now that $\mathcal{D}$ is totally geodesic. Combining this with $\left(^{*}\right)$, we have that $\mathcal{D}$ is $\nabla$-parallel on $T^{2}$. The shape operator $S$ might have different rank at different points of $T^{2}$. Observe that $S(\mathcal{D}) \subset \mathcal{D}$ (this is obvious on $M_{2}$ ). On $M_{1}$ we have $\operatorname{ker} h=\operatorname{ker} R=\mathcal{D}$. If $0 \neq X \in \operatorname{ker} R$ and $Z$ is linearly independent of $X$, then $R(X, Z) Z \neq 0$, and by the Gauss equation we get

$$
\operatorname{im} R \ni R(X, Z) Z=h(Z, Z) S X .
$$

Thus $S X \in \mathcal{D}$. We now consider the same plane field $\pi$ as in the previous case, i.e., $\pi$ is given by (3.2). By using formulas (1.1), (1.2) and the facts that $S(\mathcal{D}) \subset \mathcal{D}$ and $\mathcal{D}$ is totally geodesic, one sees that the field $\pi$ is constant along orbits of $\mathcal{D}$. Using the same formulas, the parallelism of $\mathcal{D}$ and the equality $\operatorname{im} R=\operatorname{im} S$ on $M_{2}$, we get that $\pi$ is constant on each connected component of $M_{2}$.

We shall now show that $\pi$ is locally constant on $T^{2}$. Let $x \in M_{1}$, and let $\gamma(t)$, $t \in[0, b), b \in \mathbf{R}$ or $b=\infty, \gamma(0)=x$, be a maximal geodesic in the direction of $\mathcal{D}$. Suppose $\operatorname{im} \gamma \subset M_{1}$. Since $\mathcal{D}$ is totally geodesic, $\gamma^{\prime}(t) \in \mathcal{D}$ for every $t$. For each $x \in M_{1}$ we have $\mathcal{D}_{x}=\operatorname{ker} h_{x}$. Therefore, using formula (1.1), we see that $f \circ \gamma$ is a geodesic relative to $\tilde{\nabla}$ in $\mathbf{R}^{3}$. Since $f\left(T^{2}\right)$ is compact, $b \in \mathbf{R}$. By Proposition 3.2 $f$ is extrinsically complete, and consequently $\gamma(t)$ has a limit in $T^{2}$ for $t \rightarrow b$. But this means that $\gamma$ can be extended as a geodesic beyond $b$, i.e., $\gamma$ is not maximal. Hence im $\gamma$ must contain a point from $M_{2}$. Let $y=\gamma(c) \in M_{2}$.

Around any $x \in T^{2}$ there is a coordinate system $(u, v)$ on a neighbourhood $W$ of $x$ :

$$
W=\left\{(u, v) ; a_{1}<u<a_{2}, b_{1}<v<b_{2}\right\},
$$

such that $\partial_{u}$ spans $\mathcal{D}$. Such a neighbourhood $W$ will be called $\mathcal{D}$-adapted. By a $\mathcal{D}$-adapted subneighbourhood of $W$ we shall mean $W^{\prime} \subset W$ described in the coordinate system $(u, v)$ by $\left\{\left(a_{1}, a_{2}\right) \times\left(b_{1}^{\prime}, b_{2}^{\prime}\right)\right\}$ where $\left(b_{1}^{\prime}, b_{2}^{\prime}\right) \subset\left(b_{1}, b_{2}\right)$.

Cover $\operatorname{im} \gamma=\gamma([0, c])$ by a finite number of $\mathcal{D}$-adapted neighbourhoods $W_{1}, \ldots$, $W_{k}$ of points in $\operatorname{im} \gamma$. Assume that $y \in W_{1}, W_{1} \subset M_{2}, W_{i} \cap \operatorname{im} \gamma$ is connected, and $W_{i} \cap W_{i-1}(i>1)$ contains a piece of $\operatorname{im} \gamma$. Since $\pi$ is constant on $W_{1}$ and constant along the orbits of $\mathcal{D}$, there is a $\mathcal{D}$-adapted subneighbourhood $W_{2}^{\prime}$ of $W_{2}$ on which $\pi$ is constant. Continuing in this way we obtain that there is a neighbourhood of $x$ on which $\pi$ is constant. Hence $\pi$ is constant on $T^{2}$. Let $\mathbf{e}$ be a nonzero vector of $\mathbf{R}^{3}$ perpendicular to $\pi$. At a point $x$ where the function given by (3.1) attains an extremum we have $f_{*}\left(T_{x} T^{2}\right)=\pi_{x}$. This contradicts the definition of $\pi$. The proof is completed.

\section{REFERENCES}

1. G. S. Birman and K. Nomizu, The Gauss-Bonnet theorem for 2-dimensional spacetimes, Mich. Math. J., 31 (1984) 77-81. MR 85g:53073

2. C. Godbillon, Dynamical Systems on Surfaces, Springer-Verlag, Berlin, Heidelberg, New York, 1983. MR 84b:57018 
3. J. Milnor, On the existence of a connection of curvature zero, Comment. Math. Helv., 32 (1958) 215-223. MR 20:2020

4. S. Kobayashi and K. Nomizu, Foundations of Differential Geometry, vol. II, Interscience, Wiley, New York, 1969. MR 38:6501

5. O. Kowalski, B. Opozda, and Z. Vlášek, A classification of locally homogeneous affine connections with skew-symmetric Ricci tensor on 2-dimensional manifolds, Monatshefte für Mathematik, 130 (2000), 109-125. MR 2001f:53048

6. K. Nomizu and T. Sasaki, Affine differential geometry, Cambridge University Press, Cambridge, 1994. MR 96e:53014

7. B. Opozda, Locally symmetric connections on surfaces, Results in Math., 20 (1991), 725-743. MR 93b:53014

8. B. Opozda, Curvature homogeneous and locally homogeneous affine connections, Proc. Amer. Math. Soc., 124 (1996), 1889-1893. MR 96h:53030

9. B. Opozda, Affine versions of Singer's theorem on locally homogeneous spaces, Ann. Global Anal. Geom., 15 (1997), 187-199. MR 98d:53032

10. B. Opozda, On locally homogeneous G-structures, Geom. Dedicata, 73 (1998), 215-223. MR 99k:53042

11. B. Opozda, A new cylinder theorem, Math. Ann., 312 (1998), 1-12. MR 2000j:53074

12. B. Opozda, A classification of locally homogeneous connections on 2-dimensional manifolds, to appear in J. Diff. Geom. Appl.

Instytut Matematyki Uniwersytet Jagielloński, ul. Reymonta 4, 30-059 Kraków, POLAND

E-mail address: opozda@im.uj.edu.pl 